\title{
La bataille du sucre ou la défaite méconnue de Napoléon I ${ }^{\text {er }}$
}

The battle of sugar or the unknown defeat of Napoleon I

\section{Ludovic Laloux}

\section{(2) OpenEdition}

\section{Journals}

Édition électronique

URL : http://journals.openedition.org/artefact/2674

DOI : $10.4000 /$ artefact.2674

ISSN : 2606-9245

Éditeur :

Association Artefact. Techniques histoire et sciences humaines, Presses universitaires du Midi

Édition imprimée

Date de publication : 15 mars 2019

Pagination : 35-56

ISBN : 978-2-8107-0623-5

ISSN : 2273-0753

Référence électronique

Ludovic Laloux, «La bataille du sucre ou la défaite méconnue de Napoléon ler », Artefact [En ligne], 9 |

2018, mis en ligne le 04 mars 2020, consulté le 27 novembre 2020. URL : http://

journals.openedition.org/artefact/2674 ; DOI : https://doi.org/10.4000/artefact.2674

\section{(c) (i) (9)}

Artefact, Techniques, histoire et sciences humaines est mise à disposition selon les termes de la Licence Creative Commons Attribution - Pas d'Utilisation Commerciale - Pas de Modification 4.0 International. 


\section{La bataille du sucre ou la défaite méconnue de Napoléon I ${ }^{\text {er }}$}

\section{Ludovic Laloux}

\section{Résumé}

Souvent présenté comme le promoteur du sucre de betterave dans le cadre du blocus britannique de 1806, Napoléon joue en réalité, à cet égard, dans le registre de la communication politique. En fait, le soutien originel à cette production réside dans l'appui des rois de Prusse au xvIII siècle. À l'aube du xIXe siècle, plusieurs hommes parviennent à en produire en France, en particulier à Lille, mais le pouvoir impérial les méconnaît. Bien tardivement, en mars 1811, l'Empereur s'intéresse à cette production jusque-là négligée par les autorités politiques françaises. Il charge Montalivet, ministre de l'Intérieur, de diriger une véritable campagne saccharifère mais le résultat s'avère un échec, hormis dans le BasRhin, la Meurthe, le Nord et autour de Paris. Chaptal remplace Montalivet dans cette mission l'année suivante mais sans guère plus de réussite. En 1813, précédant de quelques mois la chute de l'Empire liée aux revers militaires, la production de sucre de betterave disparaît pratiquement du pays.

\section{Mots-clés}

Achard, betterave, blocus, Chaptal, graine, Montalivet, Napoléon, sucre

99 Ludovic Laloux, «La bataille du sucre ou la défaite méconnue de Napoléon ler », Artefact, 9, 2018, p. 35-56. 


\section{The battle of sugar or the unknown defeat of Napoleon I}

\section{Abstract}

Napoleon was often portrayed as a promoter of sugar beet during the British embargo of 1806 but in reality his role could be summed up as political communication. In fact, support for sugar beet production originally came from the Prussian kings in the $18^{\text {th }}$ century. At the start of the $19^{\text {th }}$ century, some did produce sugar beet in France, especially in Lille, but the imperial rulers didn't really care. Very much later, in March 1811, the Emperor became interested in sugar beet production, which up until then had largely been ignored by the French political authorities. He charged Montalivet, Home Secretary, to run a fully-fledged sugar campaign, but it turned out to be a failure, with the exclusion of the Lower Rhine, the Meurthe, the North and around Paris. Chaptal took over the job from Montalivet the following year but was no more successful. In 1813, few months before the fall of the Empire, due to military defeat, sugar beet production nearly disappeared from the country entirely.

\section{Keywords}

Achard, beet, blockade, Chaptal, Montalivet, Napoléon, seed, sugar 
$\grave{A}$ côté d'une impressionnante série de victoires, la perte de SaintDomingue (1803) mais aussi l'échec cuisant de Trafalgar (1805), la retraite de Russie (1812) ou la déroute de Leipzig (1813) comptent au nombre des revers de Napoléon Bonaparte, avant le désastre de Waterloo en 1815. À cette liste s'ajoute une défaite méconnue de l'Empereur, la bataille du sucre ${ }^{1}$, qu'adroitement le pouvoir impérial sut maquiller en une victoire incontestée, au point de duper plusieurs générations successives. Du XVI ${ }^{\mathrm{e}}$ au XVIII ${ }^{\mathrm{e}}$ siècle, provenant du Nouveau Monde et en particulier de Saint-Domingue, les arrivages en métropole de sucre de canne connaissent un succès grandissant. Prévaut souvent l'idée que le blocus britannique instauré en 1806 aurait empêché de débarquer du sucre dans les ports français et, en réaction, donné l'idée à Napoléon d'encourager la production de sucre à partir de la betterave. Or, la première intervention de l'Empereur en ce sens date de 1811. En fait, dès 1791, la situation saccharifère s'avère plus complexe en Europe avec un effondrement des approvisionnements en sucre de canne, tandis que la France ne s'en émeut guère pendant deux décennies et alors qu’à partir de la Prusse s'organisent les premiers balbutiements de l'extraction du sucre de betterave. Dans ce contexte marqué par des rivalités internationales, comment appréhender non seulement le degré de sincérité des déclarations assez tardives de Napoléon en faveur de l'extraction du sucre de betterave, monopolisant bien des rouages administratifs et prenant des accents d'ordre de bataille, mais aussi leur portée ? En d'autres termes, quel crédit accorder aux décisions impériales alors qu'elles paraissent, surtout, correspondre à la mise en place d'une force de frappe administrative destinée à remporter des succès dans le domaine agricole par nature incertain ? De plus, les conseils prodigués pour la mise en culture d'une plante quasi inconnue se révèlent-ils à la hauteur des enjeux et, par ailleurs, qu'en est-il sur le plan technique de l'installation d'un outillage spécifique pour extraire le sucre des racines obtenues?

Dans un premier temps, à la charnière du XVIII ${ }^{\mathrm{e}}$ et du XIX ${ }^{\mathrm{e}}$ siècle, l'étude du contexte initial vise à comprendre que la dégradation des approvisionnements sucriers ne suscite que peu de réactions du pouvoir politique, sans doute obnubilé par l'ombre de Parmentier qui plaide en faveur d'une

1. Formule utilisée pour le titre d'un article qui considère la politique de Napoléon Ir en faveur de la betterave pour produire du sucre comme une réussite : Paul Ganıère, "La bataille du sucre ", Revue du souvenir napoléonien, $\mathrm{n}^{\circ} 257$, janvier 1971, p. 15-18. 
extraction du sucre à partir du raisin. Ensuite, la réaction en 1811 de Napoléon, pour se lancer dans la bataille du sucre, se révèle bien tardive par rapport au calendrier des travaux agricoles et, de ce fait, trahit une méconnaissance des questions agronomiques pour se placer dans les meilleures conditions afin de remporter la victoire. Ceci tend à expliquer le sombre bilan de Montalivet en fin d'année. Enfin, le niveau désastreux de ce rapport porté à la connaissance de l'Empereur explique que Chaptal, désormais désigné au seuil de l'année 1812 pour promouvoir l'extraction du sucre à partir de la betterave, situe sa campagne sucrière sous les auspices inavoués d'une opération de communication politique destinée à marquer les esprits, sans que ce stratagème ne puisse toutefois constituer un gage de réussite.

\section{Les prémices occultées de l'extraction du sucre de betterave}

$\mathrm{Au} \mathrm{XVIII}^{\mathrm{e}}$ siècle, les premières marques d'attention en faveur de la betterave et de son sucre proviennent du monde germanique avec les travaux d'Andreas Sigismund Marggraf couronnés par un certain succès dès $1747^{2}$, de son disciple berlinois Franz Karl Achard menés à partir de 1784 et d'autres savants allemands comme Wilhelm August Lampadius qui, de 1799 à 1804, consacrent une vingtaine de brochures au sucre de raves $^{3}$. Elles se caractérisent par des encouragements royaux prussiens et reçoivent un écho favorable de savants ou d'ingénieurs en Suède, en Russie et en Angleterre mais aussi à Vienne et à Bruxelles ${ }^{4}$. La découverte

2. Andreas Sigismund Marggraf, "Expériences chimiques faites dans le dessein de tirer un véritable sucre de diverses plantes, qui croissent dans nos contrées ", Histoire de l'Académie royale des sciences et belles-lettres (1747), t. 3, Deutsche Akademie der Wissenschaften zu Berlin, Haude et Spener, 1749, p. 79-90.

3. D'après Wilhelm Stieda, Franz Karl Achard und die Frühzeit der deutschen Zuckerindustrie, Leipzig, Hitzel, 1928.

4. Au moins trois chimistes suédois prolongent les travaux de Marggraf : Torbern Olof Bergman, Carl Leonhard Stälhammer et Joannes Afzelius Arvidsson. Voir Joannes Afzelius Arvidsson, "Alkali végétal sucré ", in Torbern Olof Bergman (dir.), Opuscules chimiques et physiques, t. 1, Dijon, Impr. Frantin, 1780, p. 270-300. En 1802, en Russie, le major-général Blankenagel fonde une fabrique de sucre à Akabef, au sud de Moscou, près de Toula. Le tsar Alexandre I ${ }^{\text {er }}$ l'encourage par un soutien financier de 50000 roubles. Voir : Journal de l'Empire, 20 juillet 1808, 4 p., p. 1; Harvey Washington WILEY, The sugar beet industry - Culture of the sugar-beet and manufacture of beet sugar, Washington, Department of Agriculture, 1890, p. 30. Des revues anglaises présentent 
de l'extraction du sucre aurait pu être facilement répandue en France en raison des recherches la concernant publiées en français, surtout à une époque où Paris et d'autres grandes villes du royaume se couvrent de salons littéraires et philosophiques dans lesquels se diffusent de nouvelles idées mais aussi des techniques novatrices, objets de discussions savantes. Tel ne fut pourtant pas le cas. Finalement, à des fins fourragères, cette racine pénètre en France grâce à la promotion assurée par la maison Andrieux \& Vilmorin ${ }^{5}$, ainsi que par l'exemple de sa mise en culture par l'abbé N. de Commerell, membre de la Société royale d'agriculture de Paris qui habite à Puttelange dans la partie germanophone de la Lorraine, qui s'en révèle un ardent propagateur ${ }^{6}$. Dans ses Voyages qui relatent ses séjours en France, Arthur Young mentionne l'action de cet abbé mais il considère que, si la betterave peut nourrir le bétail, cette opération ne peut se révéler fructueuse que « là où la main-d'œuvre est extrêmement bon marché ${ }^{7}$ ». À la différence d'autres pays européens, l'engouement en France à l'égard de cette plante s'avère finalement limité en raison d'inerties, ce qui rejoint le tiraillement analysé par André-Jean Bourde entre agronomie en action, résistances locales et pionniers de l'agriculture ${ }^{8}$.

La révolte d'esclaves à Saint-Domingue en 1791 entraîne une baisse sévère des arrivages de sucre de canne en Europe. Liées à sa cherté, les « émeutes du sucre » qui éclatent à Paris en janvier 1792 prouvent que sa consommation se répand progressivement au point que, des habitudes étant prises, il

le procédé d'Achard, la première en 1805 : «New method of extracting raw sugar from the beetroot ", The repertory of arts, manufactures and agriculture, vol. 7, n 37, 1805, p. 228-230. Achard entretient une correspondance qui contribue à la diffusion internationale de ses recherches avec des savants comme Johann Andreas Scherer à Vienne, en Autriche, et Jean-Baptiste Van Mons à Bruxelles, professeur de chimie et d'agronomie à l'université de Louvain et élu associé non résidant de l'Institut national à Paris. Voir " Note sur l'extraction du sucre de betterave, (beta vulgaris Linn.) - Extraite d'une lettre de M. A. N. Scherer au citoyen Van Mons ", Annales de chimie, t. 30, 30 germinal an VII (19 avril 1799), p. 299-302.

5. Catalogue des plantes, arbres, arbrisseaux, et arbustes, Paris, Andrieux et Vilmorin, 1778.

6. N. de Commerell, Mémoire et instruction sur la culture, l'usage et les avantages de la racine d'abondance ou de disette, Paris, Buisson, 1786. L'ouvrage connait rapidement plusieurs éditions en France et à l'étranger, notamment à Lausanne, Londres et Bouillon.

7. Arthur Young, Voyages en France en 1787, 1788 et 1789 - Le travail et la production en France : agriculture, commerce, industrie, traduction par Henri Sée, Paris, Armand Colin, 1931, p. 1157.

8. André-Jean Bourde, Agronomie et agronomes en France au XVIIt siècle, 3 vol., Paris, SEVPEN, 1967. 
devient parfois difficile de s'en passer. L'effondrement des approvisionnements ${ }^{9}$ n'en conduit pas moins à une diminution de la consommation. Le 11 janvier 1799, Achard présente au roi de Prusse Frédéric-Guillaume III des échantillons de sucre obtenu à partir de betteraves ${ }^{10}$.

Le 10 avril 1799 à Paris, au siège de l'Académie des sciences, Guyton lit à ses confrères une lettre d'Achard à Van Mons sur le procédé d'extraction du sucre de betterave suivi par ses soins pour trouver environ $6 \%$ de sucre dans cette racine ${ }^{11}$. Seulement cinq jours plus tard, Deyeux, Fourcroy et L'Héritier, membres de cette institution, livrent à leur tour un rapport à leurs pairs à propos d'un mémoire de Deslozières relatif à la production de sucre à partir de plantes qui croissent en France ${ }^{12}$. Or, à la fin de leur intervention, d'une manière assez péremptoire, ils évoquent l'usage du procédé de Marggraf et d'Achard et le cantonnent à un "laboratoire de chimie $^{13}$ ". Le 20 mai 1799, l'Institut national confie à une commission le soin d'établir un rapport comparatif pour évaluer, sur le plan économique, le procédé le plus intéressant pour extraire du sucre de diverses espèces végétales ${ }^{14}$. Le 25 juin 1800, soit treize mois plus tard, la commission rend compte de sa mission ${ }^{15}$. Son rapport commence par la présentation de la technique suivie par Achard qui consiste en un découpage des betteraves en tranches, en leur séchage puis à leur ébullition dans de l'alcool, avant de traiter le produit obtenu avec de l'alcool concentré afin d'isoler les cristaux de sucre pur. Toutefois, bien qu'informée des différentes étapes du procédé, l'équipe de savants français réitère l'expérience

\footnotetext{
9. $95000 \mathrm{t}$ en 1788 puis $25000 \mathrm{t}$ en 1807, d'après Christian Schnakenbourg, Histoire de l'industrie sucrière en Picardie (1810-2006), Paris, L'Harmattan, 2010, p. 16.

10. Voir Franz Achard, Kurze Geschichte der Beweise, welche ich von der Ausführbarkeit im Grossen und den vielen Vortheilen der von mir angegebenen Zuckerfabrication aus Runkelrüben geführt habe, Berlin, Impr. Carl Ludwig Hartmann, 1800.

11. Institut de France, Archives de l'Académie des sciences, compte rendu de la séance du 21 germinal an VII (10 avril 1799) de l'Académie des sciences.

12. Institut de France, Archives de l'Académie des sciences, Nicolas Deyeux, Antoine-François Fourcroy, Charles L'héritier, "Rapport sur le mémoire du citoyen Deslozières ", texte lu à la séance de l'Académie des sciences du 26 germinal an VII (15 avril 1799).

13. Ibid., p. 10.

14. Commission composée de Cels, Chaptal, Darcet, Deyeux, Fourcroy, Guyton, Parmentier, Tessier et Vauquelin.

15. Nicolas Deyeux, «Extrait du rapport fait à la classe des sciences mathématiques et physiques de l'Institut national [le 6 messidor an VIII (25 juin 1800)], par la commission chargée de répéter les expériences de M. Achard sur le suc de betterave, par le cit. Deyeux ", Annales de chimie, t. 35, 30 messidor an VIII (19 juillet 1800), p. 134-152.
} 
d'Achard mais sans cuire la racine, ce qui ne respecte pas la démarche suivie par ce dernier. Apprenant cette omission, le savant berlinois s'en émeut d'ailleurs ${ }^{16}$. De plus, cette commission s'attelle à la tâche dans la deuxième moitié de l'hiver 1800 avec des betteraves qui ne peuvent offrir qu'une fraîcheur altérée à ce stade de l'année. En effet, ces racines perdent de leur substance sucrée au fur et à mesure du temps, ce qu'accélèrent une aération insuffisante et le réchauffement de la température ambiante qui génère le développement de moisissures ${ }^{17}$. À l'époque, ces savants ignorent vraisemblablement ces informations, mieux connues ultérieurement, mais ceci signifie que les résultats de leurs travaux ne peuvent qu'être biaisés. Ces éléments éclairent leur approche critique à l'égard de leur collègue prussien car, à leurs yeux, son procédé d'extraction du sucre de betterave se révèle non rentable : " [...] Deyeux et Parmentier sont arrivés à des résultats qui ne permettent pas de regarder cet objet comme économique ${ }^{18}$ ". Emma Spary indique le scepticism qui prévaut à Paris à propos des travaux d'Achard $^{19}$. Le mot s'avère sans doute faible. En réalité, le rapport de la commission dirigée par Deyeux rejette les conclusions du savant berlinois. La position de l'Institut national donne le ton en France où désormais, et pour plusieurs années, domine un entrain limité à propos des recherches relatives à l'extraction du sucre de betterave.

Face à la pénurie qui s'instaure, l'attitude de Parmentier (1737-1813), farouche opposant à cette racine jusqu'à sa mort, peut étonner de la part de ce promoteur d'autres plantes, en particulier de la pomme de terre, pour laquelle il obtint l'attention et le soutien aussi bien de Louis XVI que de Napoléon, et du maïs. Parmentier développe une véritable aversion à l'égard de la betterave, critiquant Marggraf et, d'une manière générale, les partisans de l'extraction du sucre de betterave au point de la qualifier de

16. Voir « Extrait d'une lettre [du 16 novembre 1800 à Berlin] de M. Achard au citoyen Van Mons ", Annales de chimie, t. 37, 30 nivôse an IX (20 janvier 1801), p. 223.

17. Généralement, l'extraction du sucre de betterave s'effectue au cours de l'automne. En effet, selon le dicton relatif à la Saint-Luc fêtée le 18 octobre, "À la Saint-Luc, la betterave devient sucre ». Cette date illustre l'apogée de la campagne betteravière qui couvre la période de la mi-septembre à la fin décembre.

18. Jean Antoine Chaptal, Chimie appliquée aux arts, Paris, Impr. Crapelet, t. 2, 1807, p. 476.

19. Emma Spary, Feeding France - New sciences of food, 1760-1815, Cambridge, Cambridge University Press, 2014, p. 287. 
«travail de pure curiositée ${ }^{20}$ ». Pour lui, la betterave doit être réservée à l'alimentation des bestiaux car, pour cette racine, il s'agit de "l'emploi le plus utile et le plus raisonnable ${ }^{21} »$. Il préconise de s'orienter vers le raisin pour obtenir du sucre. En 1805, il invite à «augmenter en France la fabrication du raisiné, afin de diminuer pour le présent et pour l'avenir le sucre ${ }^{22}$ » de canne consommé. Toutefois, cette voie se révèle peu adéquate car, par nature, le sirop sucré obtenu à partir du raisin ne peut cristalliser. En effet, s'il contient du saccharose, sa teneur en fructose et en glucose s'avère trop importante pour permettre la cristallisation.

En ce début du XIX ${ }^{e}$ siècle, les tensions entre la France et la GrandeBretagne s'avivent et, après la rupture de la paix d'Amiens (1803), se muent en une guerre qu'illustre, en particulier, la défaite navale franco-espagnole de Trafalgar du 21 octobre 1805 face aux Britanniques. Ces derniers maitrisent alors mers et océans, ce qui les enhardit à décréter le 16 mai 1806 un blocus de tous les ports et côtes du continent européen afin de contrer l'influence française. Cette décision doit notamment empêcher les arrivages en France de sucre de canne produit dans les Antilles. En réplique, la France décrète à son tour, en novembre 1806, un blocus contre l'Angleterre. L'efficacité des mesures prises laisse songeur. En effet, la contrebande active permet des arrivages illicites de sucre en France. Fin novembre 1807, La Feuille du département du Nord, publiée «Avec permission de S.E. $\mathrm{M}^{\mathrm{gr}}$ le ministre de la police, et approbation du général préfet du département du Nord ${ }^{23}$ ", en offre une illustration par la publication d'un tableau relatif aux cours moyens constatés à Lille de produits en provenance d'outre-mer, résultant probablement de saisies effectuées dans le cadre du blocus : riz, potasse, café, coton, cacao mais aussi sucre qui, selon les mentions d'origine signalées, arrivent non seulement de la Martinique et de La Havane mais aussi de la Jamaïque, alors possession anglaise ${ }^{24}$. De son côté, Parmentier estime le sucre non nécessaire et incite à lui trouver des substituts. À cet égard, il publie en 1808 : Instruction sur les moyens de

20. Antoine Augustin PARmentier, Cours complet d'agriculture théorique, pratique, économique, et de médecine rurale et vétérinaire; ou dictionnaire universel d'agriculture, t. 11, Paris, Marchant, 1805, p. 232-236 (citation p. 236).

21. Ibid.

22. Antoine Augustin Parmentier, "De la notice historique et chronologique de la matière sucrante ", Annales de chimie, t. 10, 31 octobre 1811, p. 293-313 (citation p. 305).

23. La feuille du département du Nord, 26 novembre 1807, p. 1.

24. « Prix courant des marchandises, vendues à Lille, le 25 novembre 1807 », ibid., p. 4. 
suppléer le sucre dans les principaux usages qu'on en fait pour la médecine et l'économie domestique 25 .

Si, en 1808, Napoléon semble s'intéresser à la production saccharifère, il ne s'en montre pas moins mal informé des travaux scientifiques accomplis par Achard neuf ans plus tôt à partir de la betterave, au point d'écrire à Claude-Louis Berthollet, membre de l'Institut : «Est-il vrai qu'un nommé Achard ait fait, à Berlin, de [sic] bon sucre avec de l'érable, et qu'on puisse faire également avec des navets du sucre qui est fort bon ? Faites, je vous prie, des recherches là-dessus ${ }^{26}$. " Par la suite, l'Empereur ne paraît guère plus s'intéresser aux travaux d'Achard. Cependant, devait-il vraiment se pencher davantage sur les recherches scientifiques et techniques pour extraire du sucre à partir de la betterave alors que les blocus de 1806 se révélaient de plus en plus inopérants ? Sur ce point, Silvia Marzagalli considère que, "à partir de 1809, le système des licences rend possible une certaine reprise, légale ou presque, des trafics avec l'ennemi ${ }^{27}$. » De plus, dans un courrier adressé le 20 mars 1810 à son frère Louis Napoléon, roi de Hollande, à propos de la situation due aux deux blocus mis en place quatre ans plus tôt : "C'est une erreur de croire que la France souffre de l'état actuel. Les denrées coloniales sont en si grande quantité qu'elle ne peut pas en manquer de longtemps, et le sirop de raisin et le miel suppléent partout au sucre ${ }^{28}$. " Ainsi, les problèmes d'approvisionnement sucrier liés au blocus britannique mis en place quatre ans plus tôt ne paraissent-ils guère gênants pour l'Empereur, lui qui n'exprime alors aucunement la volonté d'y remédier.

À l'époque, de leur propre chef, plusieurs entrepreneurs français amorcent des recherches sur l'extraction du sucre de betterave : François Thierry présente ses travaux menés au cours des mois écoulés dans la Feuille de Lille

\footnotetext{
25. Antoine Augustin Parmentier, Instruction sur les moyens de suppléer le sucre dans les principaux usages quion en fait pour la médecine et l'économie domestique, Paris, Méquignon aîné, 1808.

26. “ No 13580 - À M. Berthollet, membre de l'Institut - 18 février 1808 », Correspondance de Napoléon Ier publiée par ordre de l'Empereur Napoléon III, t. 16, Paris, Plon, J. Dumaine, 1864, p. 347.

27. Silvia Marzagalli, Les boulevards de la fraude - Le négoce maritime et le Blocus continental, 18061813 - Bordeaux, Hambourg, Livourne, Villeneuve-d'Ascq, Presses universitaires du Septentrion, 1999 , p. 117.

28. « $\mathrm{N}^{\circ} 16352$ - À Louis Napoléon, roi de Hollande, à Amsterdam - 20 mars 1810 », Correspondance de Napoléon Ier publiée par ordre de l'Empereur Napoléon III, t. 20, Paris, Imprimerie impériale, 1866, p. 318-320 (citation p. 319).
} 
d'avril 1810 ; à l'automne, en cette même ville, Parsy et Crespel-Dellisse de leur côté, mais aussi Pierre Drapiez du sien, s'engagent eux aussi dans la production saccharifère. Dans ce domaine spécifique, ces Lillois contribuent par leurs talents à l'émergence d'une communauté scientifique qu'analysent, pour l'époque et à l'échelle du pays, Nicole et Jean Dhombres ${ }^{29}$. À Vandœuvre près de Nancy, Mathieu de Dombasle en est alors au stade des essais ${ }^{30}$. Le 7 novembre 1810, le préfet du Nord envoie au ministre de l'Intérieur Montalivet une lettre accompagnée d'échantillons ${ }^{31}$, offerts par F. Thierry, à propos desquels il écrit deux jours plus tard à ce dernier : "Votre sucre a la couleur, le grain, le brillant, j’ose dire même la saveur de celui des colonies ${ }^{32}$. "Dans le courrier accompagnant les échantillons, F. Thierry signale que l'un d'eux provient d'un second raffinage. Il s'agit là du produit le plus abouti qui réjouit le préfet.

Or, quelques jours plus tard, d'une manière un peu étrange pour quelqu'un qui s'était montré à plusieurs reprises fermement opposé aux travaux d'Achard, Nicolas Deyeux, pharmacien de l'Empereur, présente le 19 novembre 1810 deux pains de sucre à Paris au siège de l'Institut national ${ }^{33}$. Ce jour-là, se considérant peu compétent en la matière, il déclare s'être rapproché, pour les produire, de Jean-Pierre Barruel, chef du laboratoire de chimie de l'École de médecine, et avoir bénéficié du concours d'Allart pour le raffinage ${ }^{34}$. A posteriori, le nom de Barruel étonne quelque peu pour la phase de production du sucre brut car celui-ci n'y parvint pas par la suite et les critiques se multiplient à propos de son incapacité à tenir son rôle lorsque, nommé par l'Empereur, il dirige à Douai

29. Nicole et Jean Dhombres, Naissance d'un nouveau pouvoir : sciences et savants en France, 17931824, Paris, Payot, 1989, p. 7.

30. Le 4 décembre 1810, Mathieu de Dombasle acquiert une propriété à Vandœuvre mais ne se lance dans la culture de betteraves et une véritable production de sucre que l'année suivante. Voir Christophe Alexandre Joseph Mathieu de Dombasle, Faits et observations sur la fabrication du sucre de betteraves et sur la distillation des mélasses, Paris, Imprimeur-libraire Madame Huzard, 1822, $2^{e}$ édition ; Fabien Knittel, Agronomie et innovation - Le cas Mathieu de Dombasle (1777-1843), Nancy, Presses universitaires de Nancy, 2009.

31. Archives départementales (AD) du Nord, M 581/41A, copie de la lettre du préfet du département du Nord au ministre de l'Intérieur, 7 novembre 1810.

32. AD Nord, M 581/41A, copie de la lettre du préfet du département du Nord à François Thierry, 9 novembre 1810 .

33. Institut de France, Archives de l'Académie des sciences, "Mémoire sur l'extraction du sucre de betterave par $\mathrm{M}^{\mathrm{r}}$ Deyeux ", 19 novembre 1810.

34. Robert Allart, ancien raffineur de sucre de canne à Paris. 
(Nord) l'une des rares écoles de sucrerie. Les reproches proviennent aussi bien de ses associés, du préfet du Nord, du sous-préfet de l'arrondissement de Douai ou encore de ses étudiants ${ }^{35}$. Comme précision technique pour sa présentation de l'automne 1810, Deyeux ajoute que le produit obtenu procède d'un deuxième raffinage, comme pour les échantillons de Thierry, et qu'il se révéla impossible d'effectuer un troisième raffinage qui eût rendu le sucre plus pur en invoquant un manque de matière à raffiner ${ }^{36}$. Cette dernière remarque surprend en cette période automnale qui correspond précisément à l'époque d'arrachage des betteraves ${ }^{37}$. Néanmoins, ceci permet de couper court à toute demande de membres de l'Institut de réitérer l'expérience devant eux afin de constater, par eux-mêmes, les étapes du procédé suivi. Ces éléments invitent à s’interroger sur la réelle paternité des échantillons officiellement produits par Barruel. Il n'en demeure pas moins vrai que la question de l'extraction du sucre de betterave touche désormais le cénacle parisien proche du pouvoir impérial. Certains de ses membres y accordent désormais de l'intérêt. Ainsi, Montalivet accorde-t-il une gratification financière à Thierry pour la qualité de sa production ${ }^{38}$ et écrit-il au préfet du Nord, le 20 décembre 1810, pour lui demander

35. Ainsi, Barruel exaspère ses associés de la sucrerie Barruel \& Amet \& Fils qui déclarent : « Pour la réhabilitation dans notre esprit, pour soutenir la réputation chancelante et surtout pour vaincre notre répugnance à lui faire de nouveaux fonds, M. Barruel s'avisa, il y a un an, de nous prévenir d'une découverte superbe et magnifique qu'il venait de faire. Elle était relative au sucre de betteraves et il désirait en faire l'expérience en notre présence. M. le comte Bertholet [sic] fut témoin, comme nous de son charlatanisme [...]. » AD Nord, M 581/42, lettre d'Andriel et Wolft au ministre de l'Intérieur, 18 juillet 1813 , p. 2. Lire Berthollet.

Le préfet s'étonne de la courte durée de l'enseignement dispensé par Barruel alors que l'État prend en charge le coût de la formation des participants. Voir AD Nord, M 581/42, lettre du préfet du Nord au ministre de l'Intérieur, 23 janvier 1812, 2 p. À propos de la formation sur la production sucrière que devait dispenser Barruel, le sous-préfet constate "le mécontentement qu’a excité M. Barruel parmi les [étudiants] étrangers qui étaient venus dans l'espoir de recueillir des documents utiles pour cette nouvelle branche d'économie publique ». Voir AD Nord, M 581/42, lettre du sous-préfet de Douai au préfet du Nord, 21 mars 1812.

36. Institut de France, Archives de l'Académie des sciences, « Mémoire sur l'extraction du sucre de betterave par $\mathrm{M}^{\mathrm{r}}$ Deyeux ", 19 novembre 1810, p. 13. Au lieu de sucre plus pur, la version publiée mentionne deux pains plus beaux : Nicolas Deyeux, "Mémoire sur l'extraction du sucre de betterave; présenté à la première Classe de l'Institut national le 19 novembre 1810 », Annales de chimie, t. 77,31 janvier 1811 , p. 58.

37. Deyeux déclare avoir acquis ses betteraves à un cultivateur de Pantin. En 1810, sans être très répandue, la culture de la betterave existe à des fins fourragères depuis le siècle précédent et c'est de ces betteraves fourragères que provient le sucre de betterave.

38. AD Nord, M 581/41A, lettre du ministre de l'Intérieur au préfet du Nord, 29 novembre 1810. 
de suivre avec une extrême attention les projets d'établir sans délai trois fabriques de sucre à Lille, situation unique en France.

\section{La campagne sucrière de Napoléon en 1811 : enjeux d'une initiative tardive}

Publié le 10 janvier 1811 à propos de la production saccharifère en métropole, un rapport adressé à l'Empereur par le ministre de l'Intérieur Montalivet débute par l'évocation de la préparation possible de sirops et de sucre de raisin dans les départements méridionaux et du centre du pays ${ }^{39}$. Toutefois, dès la fin même du premier paragraphe, Montalivet oriente le texte vers les contrées septentrionales qui pourraient, selon lui, fournir un sucre de qualité à partir de la betterave. Ce document présente un véritable tournant : pour la première fois, les autorités politiques reconnaissent l'intérêt d'extraire du sucre de cette racine. Hormis la mention de la mise en culture de 80 arpents dans le Doubs permettant la production de 500 t de betteraves, information déjà donnée par Deyeux à l'Institut le 19 novembre précédent, tous les exemples fournis par Montalivet concernent des départements germaniques situés hors des frontières de la France de 1792. Montalivet se montre laudatif à l'égard de Deyeux.

En revanche, malgré les contacts au cours des semaines écoulées de novembre et décembre 1810 avec ou à propos de plusieurs d'entre eux, Montalivet ne cite pas une seule fois Thierry, Parsy, Crespel-Dellisse ou Drapiez pour leurs recherches sacchariferes actives et leurs productions prometteuses dont l'administration a connaissance. Aux alentours du 10 janvier 1811, accompagné de Deyeux, Montalivet présente à Napoléon deux pains de sucre, scène illustrée pour la postérité par Jacques-Louis David $^{40}$.

Plus de deux mois après, le 18 mars 1811, Napoléon adresse une note au ministre de l'Intérieur où il lui indique la marche à suivre pour montrer les avantages de la production du sucre de betterave et lancer la culture de

39. Voir Jean-Pierre De Montalivet, "Rapport à Sa Majesté ", Journal de l'Empire, 10 janvier 1811, 4 p., p. 3.

40. Jacques-Louis DAVID, "Le ministre de l'Intérieur présente à l'Empereur du sucre de betterave [janvier 1811] ", Guyot et Maréchal, Histoire de France sous l'Empire de Napoléon le Grand, représentée en figures, t. 6, Paris, Imprimerie Eberhart, 1813, p. 17. 
celle-ci, y compris par l'absence de taxes pendant cinq ans. Par l'évocation de la politique de prohibition du sucre de canne, il campe le décor de la promotion de celui de betterave sur fond de rivalité avec l'Angleterre. Dès le 21 mars 1811, Le Moniteur universel annonce des résultats mirifiques pour la teneur saccharifère des betteraves avec des pourcentages de 65 à $70 \%$ de sucre ${ }^{41}$, alors qu'à l'époque la betterave n'en contient qu'environ $5 \%$ et que l'extraction effective tourne autour de $1 \%$. De plus, ce journal affirme qu'après l'arrachage, les betteraves deviennent plus riches en sucre. Or, c'est l'inverse qui se produit avec une perte de l'ordre de 100 grammes par tonne de betteraves et par jour, soit une déperdition journalière de sucre d'environ $1 \%$ par rapport au sucre qu'il est alors possible d'extraire des betteraves. Pour l'époque, ces déclarations se comprennent à l'égard d'une plante mal connue mais elles n'en induisent pas moins en erreur les personnes qui envisagent de se lancer dans l'aventure sucrière indigène, suivant le qualificatif alors peu à peu adopté pour désigner la betterave cultivée pour son sucre en Europe. Enfin, le 25 mars 1811, Napoléon signe un décret afin de prendre les mesures pour approvisionner la métropole en sucre grâce à la mise en culture de 32000 ha de betteraves à travers le pays $^{42}$. L'Empereur stipule à l'administration de répartir cette superficie en fonction de la culture du tabac, en considérant que les terres favorables à celle-ci doivent l'être également pour la betterave. Le décret prévoit également l'établissement de six écoles expérimentales pour apprendre à extraire le sucre de la betterave. À l'évidence de telles mesures méconnaissent le fonctionnement sur le temps long de la vie agricole.

En effet, rédigé au seuil du printemps, ce décret ne prend pas en compte plusieurs points majeurs. Il faut des graines en quantités suffisantes réparties sur l'ensemble du territoire, ce qui nécessite de prévoir leur acheminement. De nombreux préfets soulignent les problèmes à surmonter pour s'approvisionner en semences. Celui du Gard note que le seul obstacle à ses yeux à propos des agriculteurs est " la difficulté de trouver de la graine de betterave blanche en quantité suffisante ${ }^{43} \%$. La betterave, plante bisannuelle, fleurit la première année et ne graine que la deuxième année de culture. Or, pour disposer d'un volume de graines suffisant en 1811, il aurait donc

41. "Mélanges », Le Moniteur universel, n 80, 21 mars 1811, p. 306-308 (voir p. 307).

42. Napoléon I ${ }^{\text {er }}$, décret impérial sur le sucre de betterave et le pastel (25 mars 1811), Le Moniteur universel, $\mathrm{n}^{\circ} 85,26$ mars 1811, p. 325-326.

43. AD Gard, 7 M 187, copie d'une lettre du préfet du Gard au ministre de l'Intérieur, 8 avril 1811. 
fallu en semer le volume nécessaire au début du printemps 1809, n’en récolter les graines qu'en août 1810 pour les semer à leur tour au début du printemps suivant, dans l'idée de produire du sucre en fin 1811, voire en début d'année 1812. De plus, pour une croissance optimale, les graines de betteraves doivent être semées fin mars ou début avril après de profonds labours mais, en raison des règles d'une planification administrative, la répartition des hectares selon les départements, les arrondissements et les communes prend du temps et ne s'effectue qu'au mois d'avril, voire de mai ou même plus tardivement. Ainsi, pour les 200 ha attribués à la Côte-d'Or, le préfet n'effectue que le 2 mai 1811 leur répartition entre les quatre arrondissements du département ${ }^{44}$. Il faut ensuite que les souspréfets s'adressent aux maires des communes pour ventiler les superficies à cultiver entre les paysans. De la sorte, ces derniers ne peuvent accomplir les semis qu'au moins deux mois après le décret impérial et, surtout, plus d'un mois au-delà de leur période idoine. L'inertie se révèle telle que la mise en culture de la betterave est inexistante en certains lieux. Pour expliquer cette situation dans son arrondissement, le sous-préfet de Carpentras déclare : " on a eu, un peu tard, connaissance des intentions du gouvernement ${ }^{45}$ ". Si cette remarque à propos de la planification étatique tardive s'avère juste, néanmoins d'autres raisons expliquent également le manque d'entrain à l'égard de cette racine.

Prudents par expérience, les cultivateurs veillent à effectuer un dosage entre leurs différentes cultures, notamment en semant à l'automne le blé d'hiver et vers Pâques le blé de printemps pour répartir les risques en cas de problèmes. Ceci signifie que l'ensemencement de bien des champs est déjà effectif ou en voie de l'être, avec une profondeur de labour souvent trop superficielle, dans ce deuxième cas de figure, qui ne correspond pas à la culture des betteraves lorsque les décisions de l'administration impériale arrivent au fin fond des campagnes pour sommer les paysans de produire des betteraves. Enfin, pour un grand nombre d'entre eux, il s'agit d'une plante inconnue. De ce fait, selon le sous-préfet de Sarlat, elle suscite chez les maires de son arrondissement « la plus grande répugnance ${ }^{46}$ » en raison

44. AD Côte-d'Or, M 13 VIII b 1, lettre du préfet de la Côte-d'Or au sous-préfet de Semur-enAuxois, 2 mai 1811.

45. AD Vaucluse, 6 M 345, lettre du sous-préfet de Carpentras au préfet du Vaucluse, 27 juillet $1811,2 \mathrm{p}$.

46. AD Dordogne, 7 M 25, lettre du sous-préfet de Sarlat au préfet de la Dordogne, 16 mai 1811. 
des conditions particulières qu'elle exige : elle requiert des sols drainés et des labours profonds; elle nécessite des soins spécifiques tels que le binage, le sarclage et le démariage ; il faut connaitre la date d'arrachage qu'il n'est pas évident de déterminer, de prime abord, pour des personnes ignorantes du fonctionnement même de la plante à propos du saccharose qui migre des feuilles vers la racine. De plus, un arrachage trop précoce donne des racines avec peu de sucre : en effet, plus de 2000 heures de lumière s'avèrent nécessaires pour parvenir à une concentration de sucre satisfaisante dans la racine, ce qu'il n'est guère possible de savoir avec les connaissances de l'époque. La chaleur excessive de l'été 1811 se révèle préjudiciable aux betteraves, comme l'illustre la situation de l'arrondissement de Saint-Pol-sur-Ternoise (Pas-de-Calais) où la croissance des betteraves s'y montre " languissante en raison de la sécheresse ${ }^{47}$ ». Chaude dans la France du Nord et du Centre, cette saison s'accompagne de pluies abondantes. Par rapport aux années habituelles, cette instabilité météorologique entraîne un manque de grains de $11 \%$ indique Emmanuel Le Roy Ladurie ${ }^{48}$. L'arrachage des betteraves nécessite des soins, sous peine d'endommager les racines et de rendre délicate leur conservation. Or, les champs gorgés d'eau depuis les précipitations estivales considérables reçoivent ensuite des pluies automnales importantes qui retardent ici ou là l'arrachage, ce qui perturbe les habitudes paysannes ancestrales d'équilibre dans la gestion des cultures. Les cultivateurs se trouvent alors confrontés à une situation inédite, ce qui décale le calendrier agricole avec comme conséquence le retard des labours et la difficulté de semer à temps le blé d'hiver. De la sorte, la production de betteraves peut générer des effets pervers pour d'autres cultures.

Divers problèmes surviennent encore à l'approche de l'automne quand les cultivateurs, après avoir bravé les vicissitudes administratives et les aléas agricoles, découvrent que les autorités politiques n'ont en fait rien prévu pour traiter leurs betteraves et en extraire le sucre. Dans certains départements, il existe deux ou trois fabriques de sucre, mais le plus souvent aucune : sur un total de 40 fabriques dans l'Empire, seules 25 se trouvent

47. AD Pas-de-Calais, M 1299, tableau administratif des renseignements sur la culture de la betterave et sur la fabrication du sucre de cette plante, 5 décembre 1811, p. 14.

48. Voir Emmanuel Le Roy Ladurie, Histoire humaine et comparée du climat-Disettes et révolutions 1740-1860, t. 2, Paris, Fayard, 2006. 
dans les frontières de la France de $1792^{49}$. Il faut trouver du personnel pour accomplir, avec des journées courtes et des conditions climatiques incertaines à l'approche de l'hiver, des trajets de 20,30, 40 kilomètres ou davantage pour le convoyage de charrois sur des routes ou des chemins plus ou moins carrossables. De plus, à l'avance, rien ne garantit la réception des betteraves dans la fabrique envisagée. L'incertitude demeure également grande pour le prix à en tirer. De ce fait, en Gironde, le maire de Créon interroge le sous-préfet de Bordeaux : "À quel prix les racines seront-elles achetées ${ }^{50}$ ? " Quant au fonctionnement des fabriques de sucre elles-mêmes, il faut du personnel qui maîtrise un certain savoir-faire.

Devant les difficultés rencontrées et dans le contexte de la crise économique que traverse l'Empire en 1810-1811, certains paysans rechignent à ensemencer les superficies exigées par les pouvoirs publics : en HauteGaronne, les planteurs se répartissent dans 18 communes pour un total de $7,25 \mathrm{ha}^{51}$, soit $2,42 \%$ de l'objectif des 300 ha fixé pour ce département par l'administration ${ }^{52}$. Beaucoup de cultivateurs abandonnent leurs cultures ou livrent les racines à leur bétail. En fin d'année, les résultats se révèlent décevants, hormis pour quelques départements, avec un total de $1266 \mathrm{t}$ de sucre produites, soit $39 \%$ de l'objectif ${ }^{53}$. Au vu des circonstances, il ne s'agit pas nécessairement d'un résultat si mauvais. Toutefois, Montalivet ne peut présenter un tel bilan à l'Empereur. Finalement, il compose un rapport mais son contenu idéalise la production saccharifère en métropole, ce qui irrite Napoléon. Le 30 décembre 1811, ce dernier rédige alors une note où il estime que l'année 1811 constitue un échec pour la production du sucre indigène en raison du « défaut de moyens de fabrication » conduisant à des pertes pour les cultivateurs qui « ont été dupes de leur confiance dans

49. Jean-Pierre DE MonTALivet, «Rapport à S. M. sur le sucre de betterave ", Le Moniteur universel, $\mathrm{n}^{\circ} 8,8$ janvier 1812, p. 32-34 (voir p. 32).

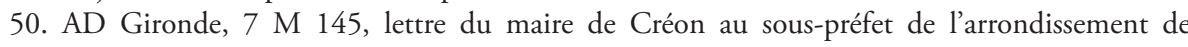
Bordeaux, 24 mai 1811.

51. AD Haute-Garonne, 9 M 20, «Tableau des renseignements sur la culture de la betterave et sur la fabrication du sucre de cette plante [en 1811] ", département de la Haute-Garonne, 1811.

52. AD Haute-Garonne, 9 M 20, lettre du ministre de l'Intérieur au préfet de Haute-Garonne, 28 mars 1811.

53. Napoléon indique un total de 2790000 livres de sucre produites, soit $1266 \mathrm{t}$, et mentionne l'objectif initial de 7200000 livres. " N 18396 - Note dictée en conseil du Commerce et des manufactures - Saint-Cloud, 30 décembre 1811 ", Correspondance de Napoléon I"r publiée par ordre de l'Empereur Napoléon III, t. 23, Paris, Henri Plon, J. Dumaine, 1868, p. 154-157 (voir p. 155). 
le gouvernement ${ }^{54}$ ». L'Empereur déplore le faible effectif de fabriques de sucre en activité et fustige Montalivet qui a affirmé jusqu'en fin d'année que leur nombre suffirait. De plus, il reproche au ministre de ne pas avoir utilisé tous les fonds mis à sa disposition. Dès lors, il exige de lui un nouveau rapport en déclarant qu'il faut masquer la réalité lors de sa publication dans le Moniteur universel. Ceci explique que, publié quelques jours plus tard en janvier, ce document ne mentionne que les statistiques relatives aux hectares de betteraves et non la production de sucre à partir de celles-ci ${ }^{55}$. Enfin, l'Empereur se tourne vers le chimiste Chaptal en lui demandant de lui fournir, pour le 6 janvier 1812, un rapport détaillé sur la production du sucre, maillon faible du processus aux yeux de Napoléon. En d'autres termes, Chaptal succède à Montalivet pour mener à bien la campagne sucrière de 1812 mais il n'a devant lui qu'une semaine pour convaincre l'Empereur du bien-fondé de son plan de bataille.

\section{Le plan de Chaptal : une opération de communication politique}

À la charnière des années 1811 et 1812, Chaptal n'a guère que quelques jours pour emporter l'adhésion de l'Empereur à ses vues. Parmi ses proches figure une connaissance de longue date, Benjamin Delessert : officier d'artillerie, tout comme Napoléon Bonaparte, au début de la Révolution française, il devient maire du $3^{\mathrm{e}}$ arrondissement de Paris en 1800 ; en 1801, il fonde une entreprise textile à Passy, commune limitrophe de Paris, et apporte son aide pour fonder la Société d'encouragement pour l'industrie nationale parrainée par Chaptal, alors ministre de l'Intérieur. Nommé régent de la Banque de France en 1802, Delessert devient membre de la Chambre de commerce en 1804 . Au seuil du XIX ${ }^{\mathrm{e}}$ siècle, il gravite ainsi dans les hautes sphères du pouvoir. Dans ses locaux à Passy, cet homme d'affaires et industriel aménage une raffinerie de sucre de canne. En décembre 1811, grâce à ses connaissances saccharifères et au talent de son ingénieur en chef Jean-Baptiste Quéruel, il se met à y produire quelques pains de sucre de betterave. Au cœur de l'hiver et alors que le temps presse, Delessert se révèle l'homme providentiel pour Chaptal. La proximité de Passy par

54. Ibid.

55. Jean-Pierre De Montalivet, « Rapport à S. M. sur le sucre de betterave ", op. cit., p. 32. 
rapport à la capitale et les liens établis entre Delessert et le pouvoir offrent à Chaptal l'occasion d'une visite inopinée, le 2 janvier 1812, de Napoléon à la fabrique de Delessert à Passy. Admiratif du travail accompli, l'Empereur lui décerne sa propre Légion d'honneur, avant de lui accorder à l'automne le titre de baron de l'Empire : de son vivant, Delessert entre dans une quasi-légende.

La réalité se révèle d'une autre nature. À partir de 5 tonnes de betteraves, Delessert est parvenu à extraire $74 \mathrm{~kg}$ de sucre. Ceci représente une extraction de sucre de 1,5\%, soit exactement le pourcentage atteint par Drapiez un an auparavant à Lille. Par ailleurs, établies à Strasbourg, les deux fabriques du Bas-Rhin, celle des frères Von der Rennen à La Robertsau et l'école de sucrerie de Maximin Isnard, produisent 292,6 t de sucre en $1811^{56}$. Ce tonnage correspond au quart de la production française. Ces entreprises alsaciennes supplantent très largement la production de Delessert mais se trouvent éloignées de Paris et des cercles du pouvoir. Il n'empêche que cette visite impromptue, aux limites de la capitale, sert de soubassement au rapport de Chaptal du 6 janvier $1812^{57}$. Quelques jours plus tard, le 15 janvier, Napoléon signe un nouveau décret qui s'en inspire et amplifie le décret rédigé dix mois plus tôt : ensemencements de 100000 ha de betteraves à des fins sucrières ; établissement de quatre fabriques impériales et de cinq écoles spéciales de chimie, pour la production du sucre de betterave, aptes à recevoir 100 élèves boursiers; octroi de 500 licences pour des fabriques de sucre exonérées de taxes. Publié deux mois plus tôt que l'année précédente, ce décret devrait permettre de gagner en efficacité.

Toutefois, des problèmes surgissent à nouveau. La hiérarchie administrative prend du retard. Ainsi, en Côte-d'Or, ce n'est que le 7 avril que le préfet envoie des kilogrammes de graines à travers tout le département ${ }^{58}$, ce qui suppose encore quelques jours de délai pour leur arrivée dans les mains des agriculteurs. Soit trois mois après le décret impérial. L'ensemencement de 100000 ha requiert 500 t de graines. Au regard de cet important volume, il faut se tourner, plus encore que l'année précédente, vers les

56. AD Bas-Rhin, 11 M 247, "Tableau de renseignements sur la culture de la betterave et sur la fabrication du sucre ", 1811.

57. Jean-Antoine Chaptal, Rapport sur la fabrication du sucre de betterave, Paris, Imprimerie impériale, 1812.

58. D'après AD Côte-d'Or, M 13 VIII b 2, lettre du préfet au sous-préfet de l'arrondissement de Châtillon-sur-Seine, 30 juillet 1812, p. 1. 
contrées germaniques ou même la Suède pour les approvisionnements. Échaudés par l'échec betteravier antérieur et par la médiocre récolte de blé en 1811 qui entraîne une hausse de son prix de vente ${ }^{59}$, des cultivateurs se montrent circonspects avant de se lancer à nouveau dans l'aventure et préfèrent plutôt subvenir aux besoins de leur famille en semant du blé pour produire du pain que de se lancer dans une culture nouvelle aux résultats aléatoires. De plus, les préparatifs de guerre " très tôt effectués ${ }^{60}$ » contre la Russie et la " machine conscriptionnelle ${ }^{61}$ » détournent sans doute une part de l'énergie des préfets nécessaire à l'application des directives sur le sucre. Enfin, dans la perspective de cette future campagne militaire, la conscription elle-même puise beaucoup dans la population paysanne. Ces éléments incitent les cultivateurs à la prudence par rapport à une innovation agronomique dévoreuse de main-d'œuvre, bien davantage que pour d'autres plantes, tout au long de la croissance de la betterave jusqu'à son arrachage.

Les 13587 ha de betteraves ensemencés en 1812 permettent la production de 1544,6 t de sucre grâce à 192 fabriques (Fig. 1). Certes la production augmente de 300 t par rapport à l'année précédente, soit $23 \%$ de plus, mais la réalité appelle des nuances. Près de la moitié des fabriques de sucre recensées concernent en fait celui de canne qui n'est alors pas d'actualité ${ }^{2}$, comme l'illustre l'effectif record de trente-trois fabriques dénombrées en Gironde qui, au total pour une campagne sucrière de trois mois, produisent 10 t de sucre. Ceci représente une moyenne de $90 \mathrm{~kg}$ par fabrique durant les trois mois de campagne betteravière, ce qui revient à un kilogramme produit par fabrique et par jour. Il est bien peu probable que ces trente-trois fabriques aient été réellement toutes en activité. Par rapport à la planification étatique de 100000 ha, seuls 13600 ha furent réellement ensemencés, soit un huitième de l'objectif. Hors du Bas-Rhin, de la Meurthe, du Nord et de la Seine, l'ampleur de la publicité d'État et des moyens déployés pour inciter à la culture de la betterave masque un échec cinglant.

\footnotetext{
59. Soit un doublement du prix du blé de 1810 à 1812 : Catherine RolLET, «L'effet des crises économiques du XIX ${ }^{\mathrm{e}}$ siècle sur la population ", Revue d'histoire moderne et contemporaine, t. 17, $\mathrm{n}^{\circ} 3$, juillet-septembre 1970, p. 396.

60. Jacques-Olivier Boudon, Napoléon et la campagne de Russie 1812, Paris, Armand Colin, 2012. 61. Annie CrépIn, "1812, fin d'une époque de la conscription ", Revue historique des armées, $\mathrm{n}^{\circ} 267,2012$, p. 33-42.

62. D'après Archives nationales (Pierrefitte-sur-Seine), F 20/560, "Tableau de l'étendue de la culture des betteraves, en 1812, indiquant le nombre de sucreries établies à cette époque et la quantité approximative de leurs produits ", statistiques gouvernementales, 1813.
} 


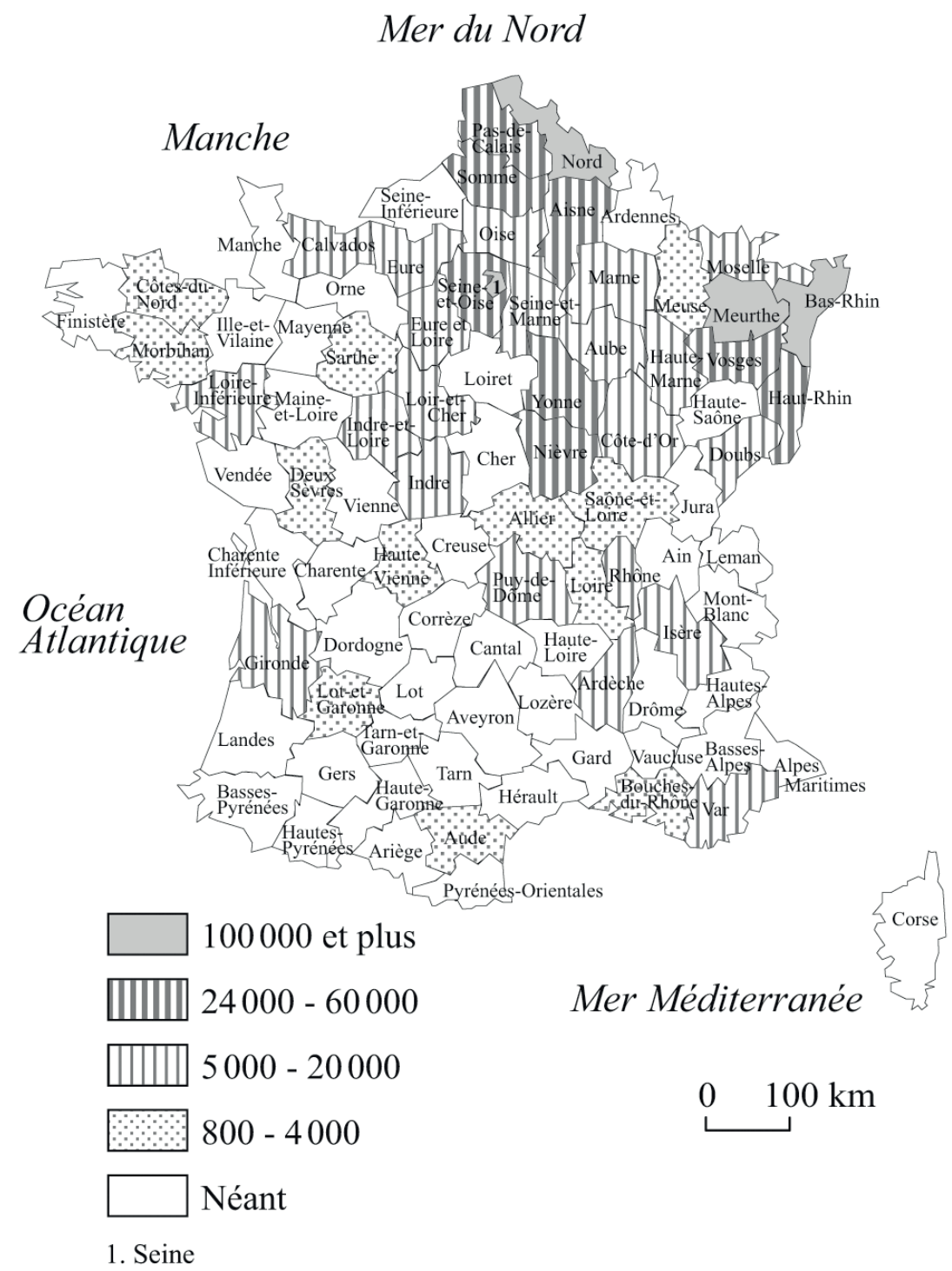

Fig. 1. - Nombre de kilogrammes de sucre indigène produit en 1812 Source : d'après Archives nationales (Pierrefitte-sur-Seine), F 20/560, « Tableau de létendue de la culture des betteraves, en 1812, indiquant le nombre de sucreries établies à cette époque et la quantité approximative de leurs produits », statistiques gouvernementales, 1813 . 
À l'achèvement de 1812, à la différence de l'année précédente, Napoléon ne relance pas sa politique sucrière. Il est vrai que l'échec de la campagne de Russie a entraîné un bouleversement de la situation militaire, politique et économique. En 1813, seul le département du Nord poursuit sa progression pour la culture de la betterave $e^{63}$, en particulier dans les arrondissements de Lille et de Douai. L'extraction du sucre s'y effectue grâce à treize fabriques, tandis que l'élan sucrier indigène s'effondre dans le reste du pays.

\section{Conclusion}

Les revers militaires qui se succèdent depuis la défaite de Leipzig à la mi-octobre 1813 conduisent à l'abdication de l'Empereur au début du printemps 1814, ce qui entraîne la fin du blocus. Dès lors, le sucre de canne arrive à nouveau dans les ports français et la production de sucre de betterave cesse pratiquement en métropole. Depuis déjà plusieurs mois, la bataille du sucre menée par le pouvoir politique au cœur du pays n'était plus d'actualité. Résultat d'une campagne aux allures d'opération de communication politique, le bilan sucrier, assez calamiteux eu égard aux moyens déployés, anticipait la chute de l'Empire. Au lieu de répartir les graines et les emblavements de betteraves à travers tout le pays suivant le schéma d'une planification étatique, sans doute aurait-il été plus judicieux de regrouper les hectares cultivés en fonction de sols adéquats et de veiller à l'édification, à proximité de ces terres, d'une fabrique de sucre afin de limiter le convoyage fastidieux de tombereaux de betteraves l'hiver venu. En 1813, le sous-préfet de l'arrondissement d'Arras rédige un avis circonstancié pour soutenir la protestation d'un Arrageois contre des pollutions et diverses dégradations attribuées à la fabrique de sucre de Philippe Ledru qui jouxte sa demeure. Le fonctionnaire recommande "d'éloigner sans délai ${ }^{64}$ » le matériel de production pour des nuisances en réalité non imputables à cette entreprise ${ }^{65}$. La mauvaise foi du représentant

63. "Culture de la betterave [1813] ", Annuaire statistique du département du Nord - 1814, p. $187-188$.

64. AD Pas-de-Calais, M 1294, avis du baron de Montigny (sous-préfet de l'arrondissement d'Arras) au préfet du Pas-de-Calais relatif à la plainte de Marie-Louis Boussemart déposée contre la fabrique de sucre de Philippe Ledru à Arras, 24 juillet 1813.

65. AD Pas-de-Calais, M 1294, François de Gantès (conseiller de préfecture), rapport pour vérifier le fonctionnement de la fabrique de sucre Ledru à Arras, 3 septembre 1813. 
de l'État étonne, d'autant plus qu'il aurait pu se montrer encourageant et bienveillant à l'égard de cet entrepreneur si désireux de répondre aux aspirations impériales. Ironie de l'histoire, de cette fabrique, reprise par Louis Crespel-Dellisse en 1815, s'opère l'impulsion salutaire au redémarrage de la production française de sucre de betterave sous la Restauration qui, avec 2665 t en 1828, dépasse pour la première fois les 1544,6 t de $1812^{66}$.

\section{L'auteur}

Maître de conférences en histoire contemporaine (habilité à diriger des recherches) à l'université de Bordeaux et membre du Centre de recherche sur les économies, les sciences, les arts et les techniques (CRÉSAT) de l'université de Haute-Alsace, Ludovic Laloux a soutenu en 2016, pour son habilitation, un mémoire inédit intitulé : La betterave à sucre : essor agricole et industrialisation rurale - Réalités françaises et perspectives internationales : de Marggraf à Berlin (1747) à la conférence de Londres (1937).Contact : Lclaloux@gmail.com

66. Voir Charles-Édouard RoYer, "Betteraves ", Notes économiques sur l'administration des richesses et la statistique agricole de la France, Paris, Imprimerie Pillet aîné, 1843, p. 342. Ce nouveau démarrage de la culture de la betterave contribue, à côté d'autres plantes sarclées, à remplacer progressivement la jachère au XIX ${ }^{e}$ siècle. Voir Pierre Morlon, François Sigaut, La troublante histoire de la jachère : Pratiques des cultivateurs, concepts de lettrés et enjeux sociaux, Versailles, Éditions Quae, 2008, p. 73. 